\title{
MACROPARTICLE SCREENING IN A WEAKLY IONIZED PLASMA
}

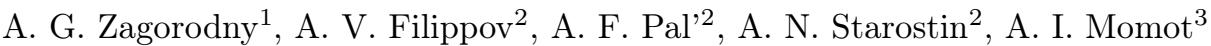 \\ ${ }^{1}$ Bogolyubov Institute for Theoretical Physics, Kyiv, Ukraine \\ ${ }^{2}$ Troitsk Institute for Innovation and Fusion Research, Troitsk, Russia \\ ${ }^{3}$ Kyiv National Taras Shevchenko University, Kyiv, Ukraine
}

(Received 29 January, 2007)

\begin{abstract}
A new approach is proposed for the analytical description of effective grain potentials in dusty plasmas. The basic idea is to describe absorption of electrons and ions by the macroparticle in terms of effective point sinks introduced into the equations of plasma dynamics. The proposed approach makes it possible to find explicit relations for the potential and particle densities distributions near the grain. It is shown that plasma particle absorption results in the long-range asymptotics of the effective grain potentials. The dynamical screening of the moving dust particle is considered as well. The energy of the grain interaction with the induced charge produced by the grain and the force acting on the moving dust particle are calculated. The possibility of negative friction for the moving grain is shown.
\end{abstract}

Key words: dusty plasma, effective potential, charge screenjng.

PACS number(s): 52.27.Lw

\section{INTRODUCTION}

The problem of nonlinear grain screening still remains one of the most important issues of dusty plasma theory. It is of great importance for the description of such interesting phenomena as dusty crystal formation, excitation of dust acoustic waves, evolution of dust structures, etc. In spite of the fact that the screened potentials have been studied for years the problem of macroparticle screening still requires further studies. First of all this concerns grain screening in low-temperature plasma for which even the screening length is not determined in the general case [1]. The problem is that in the case of grain charging due to the absorption of electrons and ions from plasma the effective potentials are crucially dependent on the processes in plasma background, in particular, on the details of plasma regeneration. In view of the importance of nonlinear effects and self-consistent description of charging processes the problem of grain (probe) screening is usually solved numerically [2-9]. Giving many details of the screening numerical results, however, cannot be used for obtaining approximate analytical relations. At the same time it is very important to have such analytical approximations for the solution of the problems mentioned above.

One more important problem is the description of the moving grain screening. Such a description is necessary for the solution of many interesting problems of dusty plasma physics, in particular, for the understanding of the physical mechanisms of energy exchange between the grain and surrounding plasma leading to the anomalous grain heating $[10,11]$. As will be shown below the model of the point sinks can be applied to the solution of this problem as well.

The purpose of the present paper is to work out an analytical description of the screened potentials of grains (the case of the moving grain included) with regard to their charging by plasma currents and to study the effects produced by grain charging on the properties of effective potentials.

We start from the formulation of the basic model (point-sink model) for the simplest case of the immovable grain. This model provides the analytical solution of the problem, if the grain charge and the intensities of plasma particle fluxes are known (Section II). Then we perform necessary analysis of the obtained relations and compare the results with the numerical solutions (Section III). In Section IV we apply the proposed model for treatment of screening of the moving grain. The results of calculations of the grain-plasma interaction energy and the electric force acting on the moving grain are presented in Section V.

\section{POINT-SINK-MODEL. BASIC SET OF EQUATIONS FOR IMMOVABLE GRAIN}

Let us consider single grain embedded into infinite weakly-ionized plasma. We assume that the grain absorbs all the encountered electrons and ions. In the stationary case the grain charge is maintained by electron and ion fluxes which are equal to each other. In the case under consideration plasma dynamics can be described by the continuity equations which have the following form:

$$
\operatorname{div} \boldsymbol{\Gamma}_{\sigma}(r)=I_{0}-\beta n_{e}(r) n_{i}(r)
$$

where

$$
\boldsymbol{\Gamma}_{\sigma}(r)=-e_{\sigma} \mu_{\sigma} n_{\sigma}(r) \nabla \Phi(r)-D_{\sigma} \nabla n_{\sigma}(r),
$$

$\mu_{\sigma}$ is the plasma particle mobility, $D_{\sigma}$ is the diffusion coefficient, subscript $\sigma(\sigma=e, i)$ labels plasma particle species, $I_{0}$ is the intensity of ionization sources, if present, $\beta$ is the coefficient of the electron-ion recombination, the rest of notation is traditional.

The electric potential $\Phi(\mathbf{r})$ satisfies the Poisson equation 


$$
\Delta \Phi(r)=-4 \pi \sum_{\sigma} e_{\sigma} n_{\sigma}(r) .
$$

Eqs. (1)-(3) should be supplemented by the following boundary conditions

$$
\begin{aligned}
\left.n_{\sigma}(r)\right|_{r=a} & =0, & \left.\frac{\partial \Phi(r)}{\partial r}\right|_{r=a}=-\frac{q}{a^{2}}, \\
\left.n_{\sigma}(r)\right|_{r \rightarrow \infty} & =n_{\sigma}, & \left.\Phi(r)\right|_{r \rightarrow \infty}=0,
\end{aligned}
$$

where $q$ is the stationary grain charge which is determined by the equation

$$
e_{i} \Gamma_{i}+\left.e_{e} \Gamma_{e}\right|_{r=a}=0,
$$

$a$ is the grain radius, $n_{\sigma}$ is the unperturbed density of particles of $\sigma$ species. The later quantities are determined by the conditions of plasma regeneration. They are described by the relation

$$
n_{e}=n_{i}=\sqrt{I_{0} / \beta} \equiv n_{0},
$$

if external sources of ionization are present, or they are assumed to be given, if the sources of plasma regeneration are located at a large distance from the grain.

In view of the nonlinearity of the basic set of equations it is usually solved numerically (see, for example, Refs. $[2,3,5-9])$. However, it is quite difficult to obtain analytical relations for the screened potentials and plasma particle distribution on the basis of the numerical solutions. Therefore, it would be highly desirable to have approximate analytical expressions for such quantities, at least for their asymptotic behavior. This problem can be solved taking into account that in many cases nonlinearity produces considerable effects at small distances from the grain surface, only. Such a conclusion follows from the analysis of the nonlinear numerical solutions for equilibrium screening (no particle fluxes through the grain surface [12]) and for the screening of grains charged by plasma currents in the collisionless plasmas [4]. Moreover, in the case of grains of small sizes the nonlinearity does not lead to a considerable deviation from the linear solution even in the vicinity of the grain, but on the other hand, the plasma particle fluxes toward the grain contribute to the changes of the potential asymptotics [4]. Probably, it could be explained by the fact that in the case of grain absorbing plasma particles the electron and ion densities near the grain are small and thus the influence of nonlinearity is not well pronounced. The possibility of using linearized equation for the analytic description of the asymptotic behavior of the effective grain potentials in weakly-ionized plasma was also confirmed by the estimates presented in Refs. [13,14].

With regard to these arguments we propose to describe screened potentials on the basis of the linearized version of the point-sink model. In terms of such a model it is assumed that the effects associated with plasma particle absorption by the finite-size grain can be approximated in a satisfactory fashion by the effective point sinks, i. e. instead of Eq. (1) and the boundary conditions (4), (5) we propose to use the equation

$$
\operatorname{div} \boldsymbol{\Gamma}_{\sigma}(r)=I_{0}-\beta n_{e}(r) n_{i}(r)-S_{\sigma} \delta(\mathbf{r}),
$$

where $S_{\sigma}$ is the intensity of the point sink

$$
S_{\sigma}=-\oint d s \boldsymbol{\Gamma}_{\sigma}
$$

The linearized version of Eqs. (2) and (7) is as follows

$$
\begin{gathered}
\frac{e_{\sigma} n_{0}}{T_{\sigma}} \Delta \Phi+\Delta \delta n_{\sigma}=\frac{\beta n_{0}}{D_{\sigma}}\left(\delta n_{e}+\delta n_{i}\right)+\frac{S_{\sigma}}{D_{\sigma}} \delta(\mathbf{r}) \\
\Delta \Phi=-4 \pi\left(e_{e} \delta n_{e}+e_{i} \delta n_{i}\right)-4 \pi q \delta(\mathbf{r}),
\end{gathered}
$$

where $\delta n_{\sigma}(r)$ is the density perturbation. We also take into account that in the absence of the grain the plasma density is given by Eq. (6) and the sink intensities for electrons and ions are equal to each other, i. e.

$$
S_{e}=S_{i}=S .
$$

\section{SCREENED POTENTIAL OF IMMOVABLE GRAIN AND CHARGE DENSITY DISTRIBUTION}

The solution of Eqs. (9), (10) can be easily obtained in the $\mathbf{k}$-representation

$$
\begin{aligned}
\delta n_{i \mathbf{k}}=\frac{1}{e \Delta}[ & -k^{2}\left(\tilde{S}_{i}+k_{D i}^{2} q\right)-q k_{0}^{4} \\
& \left.-\tilde{S}_{i} k_{D e}^{2}-\tilde{S}_{e} k_{D i}^{2}\right], \\
\delta n_{e \mathbf{k}}=\frac{1}{e \Delta}[ & -k^{2}\left(\tilde{S}_{e}-k_{D e}^{2} q\right)+q k_{0}^{4} \\
& \left.-\tilde{S}_{i} k_{D e}^{2}-\tilde{S}_{e} k_{D i}^{2}\right],
\end{aligned}
$$

where

$$
\begin{aligned}
& e=\left|e_{e}\right|=e_{i} ; \\
& \Delta=k^{4}+k^{2}\left(k_{D}^{2}+k_{S}^{2}\right)+2 k_{0}^{4} ; \\
& k_{0}^{4}=k_{S e}^{2} k_{D i}^{2}+k_{S i}^{2} k_{D e}^{2} ; \quad k_{S}^{2}=k_{S i}^{2}+k_{S e}^{2} ; \\
& k_{S \sigma}^{2}=\frac{\beta n_{0}}{D_{\sigma}}, \quad \tilde{S}_{\sigma}=\frac{e S}{D_{\sigma}} \\
& k_{D}^{2}=k_{D e}^{2}+k_{D i}^{2} \quad k_{D \sigma}^{2}=\frac{4 \pi e_{\sigma}^{2} n_{\sigma}}{T_{\sigma}} .
\end{aligned}
$$

The potential $\Phi_{\mathbf{k}}$ is given by

$$
\Phi_{\mathbf{k}}=4 \pi \frac{q\left(k^{2}+k_{S}^{2}\right)-k_{D}^{2} \tilde{S}}{k^{4}+k^{2}\left(k_{D}^{2}+k_{S}^{2}\right)+2 k_{0}^{4}},
$$

where 


$$
\tilde{S}=\frac{e S}{k_{D}^{2}}\left(\frac{1}{D_{i}}-\frac{1}{D_{e}}\right) .
$$

In its turn Eq. (15) can be rewritten as

$$
\Phi_{\mathbf{k}}=\frac{4 \pi Q_{1}}{k^{2}+k_{1}^{2}}-\frac{4 \pi Q_{2}}{k^{2}+k_{2}^{2}}
$$

where

$$
\begin{aligned}
Q_{1,2} & =\frac{q\left(k_{1,2}^{2}-k_{S}^{2}\right)+k_{D}^{2} \tilde{S}}{k_{1}^{2}-k_{2}^{2}} \\
k_{1,2}^{2} & =\frac{1}{2}\left(k_{D}^{2}+k_{S}^{2} \pm \sqrt{\left(k_{D}^{2}+k_{S}^{2}\right)^{2}-8 k_{0}^{4}}\right) .
\end{aligned}
$$

In the coordinate representation

$$
\Phi(\mathbf{r})=Q_{1} \frac{e^{-k_{1} r}}{r}-Q_{2} \frac{e^{-k_{2} r}}{r},
$$

i. e. the effective potential is given by the superposition of two screened potentials with different screening lengths. Notice that these screening lengths were obtained for the first time in Ref. [13]. At large distances the potential is determined by the term with a larger screening length. This result is in agreement with the numerical simulation [5-8].

The induced charge density is

$$
\rho(\mathbf{r})=\tilde{Q}_{1} \frac{e^{-k_{1} r}}{r}-\tilde{Q}_{2} \frac{e^{-k_{2} r}}{r} .
$$

Here

$$
\tilde{Q}_{1,2}=\frac{2 q k_{0}^{4}-k_{1,2}^{2} k_{D}^{2}(q+\tilde{S})}{k_{1}^{2}-k_{2}^{2}} .
$$

If the plasma sources are located at a large distance from the grain and recombination processes could be neglected $(\beta=0)$

$$
\Phi(r)=(q+\tilde{S}) \frac{e^{-k_{D} r}}{r}-\frac{\tilde{S}}{r}
$$

and

$$
\rho(r)=-k_{D}^{2}(q+\tilde{S}) \frac{e^{-k_{D} r}}{4 \pi r} .
$$

As is seen, in the case under consideration the effective potential has the Coulomb-like asymptotics, but the induced charge is determined by the screened part. The quantity $\tilde{S}$ can be treated as an effective charge which generates the Coulomb part of the potential. It cannot be found within the present theoretical treatment. Therefore, in what follows we take this quantity from the numerical solution (see Fig. 1), which is taken from the numerical data obtained in Ref. [6], assuming that $\tilde{S}$ is proportional to real grain charge, i. e. $\tilde{S}=-\alpha q$.

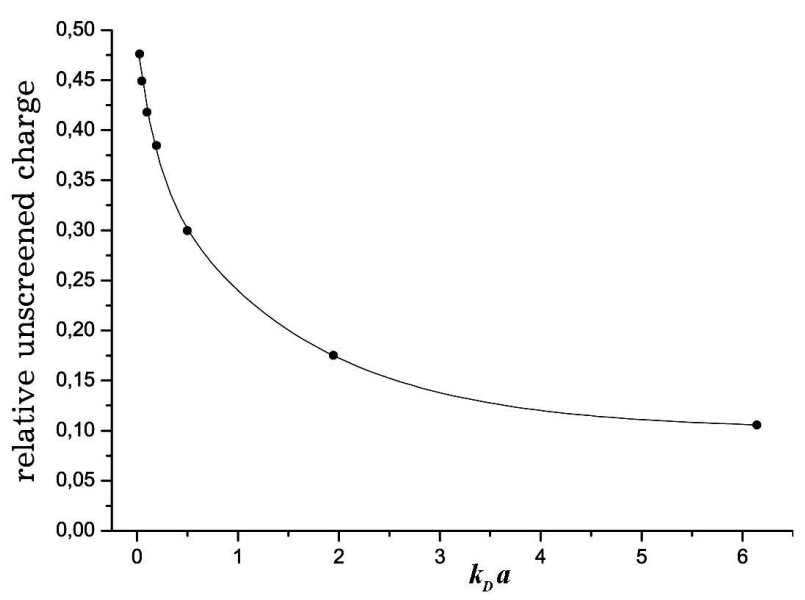

Fig. 1. Dependence of dimensionless effective unscreened charge $\tilde{Q} / q$ on dimensionless grain radius $k_{D} a$.

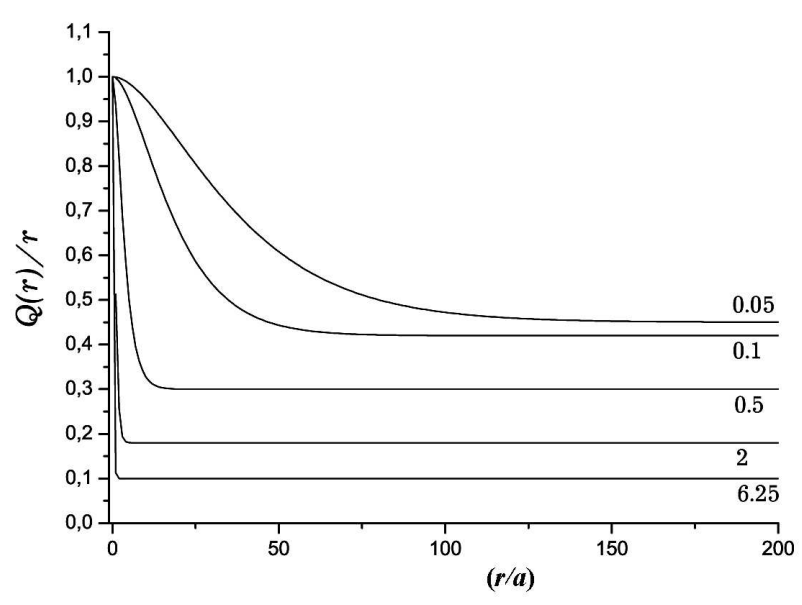

Fig. 2. Relative charge distribution corresponding to Eq.(25), at various values of $k_{D} a(0.05,0.1,0.5,2,6.25)$.

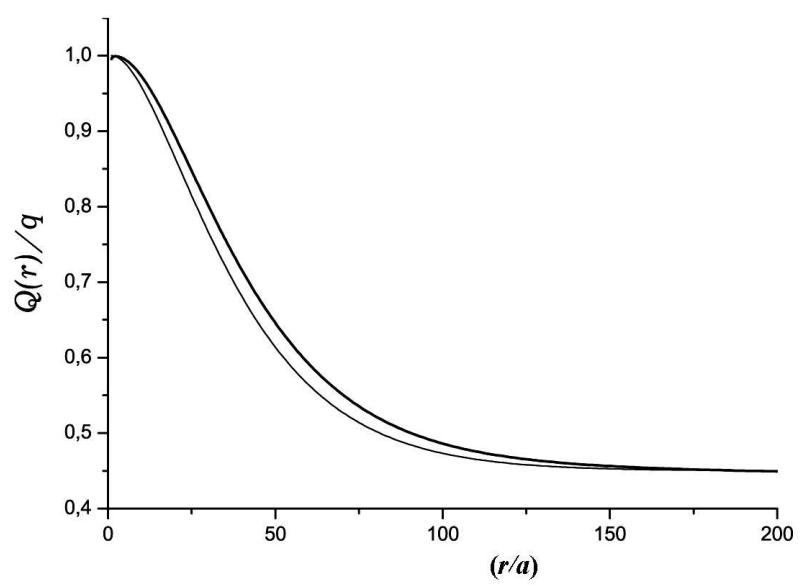

Fig. 3. Relative charge distribution at $k_{D} a=0.05$; bold line presents the data from [6], thin line corresponds to Eq. (25). 


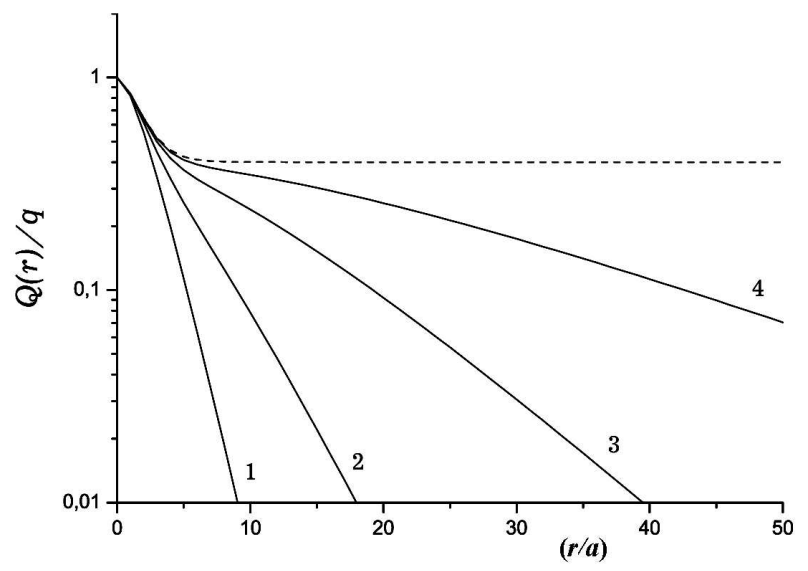

Fig. 4. Relative charge distribution at $k_{D} a=0.158$ and various values of $i_{0}=I a^{5} / D_{i}$ (1) $1.15 \cdot 10^{-2}$, (2) $2.5 \cdot 10^{-3}$, (3) $5 \cdot 10^{-4},(4) 10^{-4}$, dashed line corresponds to the case of absence of bulk ionization and recombination.

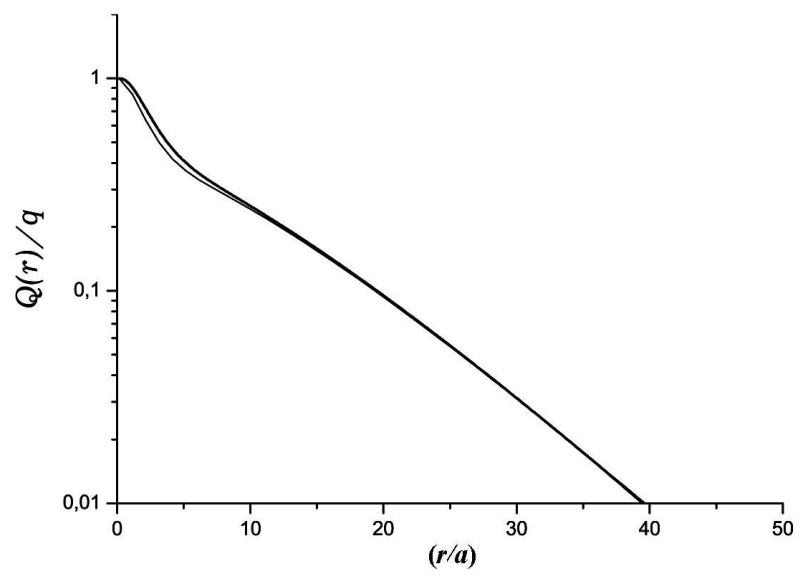

Fig. 5. Relative charge distribution at $k_{D} a=0.158$ and $i_{0}=5 \cdot 10^{-4}$, bold line presents the data from [6], thin line corresponds to Eq. (26).

To compare the obtained results with the numerical simulation [6] it is convenient to use the following quantity

$$
Q(r)=q+\int_{0}^{r} d \mathbf{r}^{\prime} \rho\left(r^{\prime}\right)
$$

which is the total charge distributed in the sphere of the radius $r$. Substitution of Eq. (23) into Eq. (24) gives

$$
Q(r)=\alpha q+q(1-\alpha) e^{-k_{D} r}\left(1+k_{D} r\right) .
$$

The dependences of the quantity $Q(r)$ on the dimensionless distances $r / a$ for different grain sizes are presented in Fig. 2. They are in good agreement with those obtained in Ref. [6]. The accuracy of the analytical estimates is seen from Fig. 3 in which the charge distributions $Q(r)$ calculated analytically (thin curve) and numerically (bold curve) are presented. As is seen, in the case under consideration $\left(\beta=0, I_{0}=0\right)$ at large distances $Q(r)$ approaches some constant values which are nothing but the dimensionless effective charge $\alpha$.

The situation is considerably changed, if the ionization sources are present. In this case

$$
\begin{aligned}
Q(r) & =q+\frac{\tilde{Q}_{1}}{k_{1}^{2}}\left[1-e^{-k_{1} r}\left(1+k_{1} r\right)\right] \\
& -\frac{\tilde{Q}_{2}}{k_{2}^{2}}\left[1-e^{-k_{2} r}\left(1+k_{2} r\right)\right] .
\end{aligned}
$$

Dependences of this quantity on the dimensionless distance for the different values of ionization intensity are presented in Fig. 4. In this case the analytical estimates are also in good agreement with the exact solution of the nonlinear equations (see Fig. 5).

\section{SCREENING OF MOVING GRAIN}

In the case of the moving grains the basic set of equations can be written as

$$
\begin{gathered}
\frac{\partial n_{\sigma}(\mathbf{r}, t)}{\partial t}-\operatorname{div} \boldsymbol{\Gamma}_{\sigma}(\mathbf{r}, t)=I_{0} \\
-\beta n_{e}(\mathbf{r}, t) n_{i}(\mathbf{r}, t)-S_{\sigma} \delta(\mathbf{r}-\mathbf{v} t), \\
\Delta \Phi(\mathbf{r}, t)=-4 \pi \sum_{\sigma} e_{\sigma} n_{\sigma}(\mathbf{r}, t)-4 \pi q \delta(\mathbf{r}-\mathbf{v} t),
\end{gathered}
$$

where $\mathbf{v}$ is the velocity of the grain. We assume that in the course of motion this velocity is not considerably changed and thus, $q$ and $S_{\sigma}$ can be treated as constants and $S_{e}=S_{i}=S$.

The linearization of Eqs. (27), (28) with respect to perturbations produced by the presence of the grain gives

$$
\begin{gathered}
\frac{\partial \delta n_{\sigma}(\mathbf{r}, t)}{\partial t}-D_{\sigma} \frac{e_{\sigma} n_{0}}{T_{\sigma}} \Delta \Phi(\mathbf{r}, t)-D_{\sigma} \Delta \delta n_{\sigma}(\mathbf{r}, t) \\
=-\beta n_{0}\left(\delta n_{i}(\mathbf{r}, t)+\delta n_{e}(\mathbf{r}, t)\right)-S \delta(\mathbf{r}-\mathbf{v} t), \\
\Delta \Phi(\mathbf{r}, t)=-4 \pi\left(e_{e} \delta n_{e}(\mathbf{r}, t)+e_{i} \delta n_{i}(\mathbf{r}, t)\right) \\
-4 \pi q \delta(\mathbf{r}-\mathbf{v} t) .
\end{gathered}
$$

The solution of these equations in $\mathbf{k}, \omega$-representation has the form

$$
\begin{aligned}
& \Phi_{\mathbf{k} \omega}=\frac{4 \pi q_{\omega}}{k^{2}}+\frac{4 \pi}{k^{2} D(\mathbf{k}, \omega)}\left\{i \omega q_{\omega}\left(\frac{k_{D i}^{2}}{D_{e}}+\frac{k_{D e}^{2}}{D_{i}}\right)\right. \\
& \left.-2 q_{\omega}\left(k_{S e}^{2} k_{D i}^{2}+k_{S i}^{2} k_{D e}^{2}\right)-k^{2}\left(q_{\omega} k_{D}^{2}+\tilde{S}_{i \omega}-\tilde{S}_{e \omega}\right)\right\}
\end{aligned}
$$

where

$$
\begin{aligned}
\tilde{S}_{\sigma \omega} & =\frac{e S}{D_{\sigma}} 2 \pi \delta(\omega-\mathbf{k v}) \\
q_{\omega} & =2 \pi q \delta(\omega-\mathbf{k v})
\end{aligned}
$$




$$
\begin{aligned}
D(\mathbf{k}, \omega) & =-\frac{\omega^{2}}{D_{i} D_{e}}-\frac{i \omega}{D_{e}}\left(k_{D i}^{2}+k^{2}+k_{S i}^{2}\right)-\frac{i \omega}{D_{i}}\left(k_{D e}^{2}+k^{2}+k_{S e}^{2}\right) \\
& +k^{4}+k^{2}\left(k_{D}^{2}+k_{S}^{2}\right)+2\left(k_{S e}^{2} k_{D i}^{2}+k_{S i}^{2} k_{D e}^{2}\right) .
\end{aligned}
$$

For the sake of simplicity in what follows we disregard the ionization and recombination processes $(\beta \rightarrow 0)$. In this case Eqs. (32), (34) reduce to

$$
\Phi_{\mathbf{k} \omega}=\frac{8 \pi^{2} \delta(\omega-\mathbf{k v})}{k^{2} D_{0}(\mathbf{k}, \omega)}\left\{i \omega q\left(\frac{k_{D i}^{2}}{D_{e}}+\frac{k_{D e}^{2}}{D_{i}}\right)-k^{2} k_{D}^{2}(q+\tilde{S})\right\}+\frac{8 \pi^{2} q \delta(\omega-\mathbf{k v})}{k^{2}} .
$$

Here,

$$
D_{0}(\mathbf{k}, \omega)=-\frac{\omega^{2}}{D_{i} D_{e}}-i \omega\left(\frac{k_{D i}^{2}}{D_{e}}+\frac{k_{D e}^{2}}{D_{i}}\right)-i \omega k^{2}\left(\frac{1}{D_{e}}+\frac{1}{D_{i}}\right)+k^{4}+k^{2} k_{D}^{2}
$$

and $\tilde{S}$ is given by Eq. (16).

The second term in Eq. (35) is the potential of the point particle with the charge $q$ in the absence of the surrounding plasma, while the first term is the induced potential.

The inverse Fourier-transformation of Eq. (35) gives

$$
\Phi(\mathbf{r}, t)=\frac{1}{2 \pi^{2}} \int \frac{d \mathbf{k}}{k^{2}} e^{i \mathbf{k}(\mathbf{r}-\mathbf{v} t)}\left\{q+\frac{i \mathbf{k v} q\left(\frac{k_{D i}^{2}}{D_{e}}+\frac{k_{D_{e}}^{2}}{D_{i}}\right)-k^{2} k_{D}^{2}(q+\tilde{S})}{k^{4}+k^{2} k_{D}^{2}-\frac{(\mathbf{k v})^{2}}{D_{e} D_{i}}-i \mathbf{k v}\left(\frac{k_{D i}^{2}}{D_{e}}+\frac{k_{D e}^{2}}{D_{i}}\right)-i \mathbf{k v v} k^{2}\left(\frac{1}{D_{e}}+\frac{1}{D_{i}}\right)}\right\} .
$$

In the case of nonabsorbing macroparticle $(S=0)$ Eq. (37) reduces to the potential of point particle moving in weakly-ionized plasma

$$
\Phi(\mathbf{r}, t)=\frac{q}{2 \pi^{2}} \int d \mathbf{k} e^{i \mathbf{k}(\mathbf{r}-\mathbf{v} t)} \frac{1}{k^{2} \varepsilon(k, \mathbf{k v})},
$$

where

$$
\varepsilon(\mathbf{k}, \omega)=1+\sum_{\sigma} \frac{i k_{D \sigma}^{2} D_{\sigma}}{\omega+i k^{2} D_{\sigma}}
$$

\section{ENERGY OF GRAIN-PLASMA INTERACTION AND DYNAMICAL FRICTION FORCE}

The energy of macroparticle interaction with the induced charge is

$$
U(\mathbf{v})=q \lim _{\mathbf{r} \rightarrow \mathbf{v} t} \Phi_{\text {ind }}(\mathbf{r}, t)=\frac{q}{2 \pi^{2}} \int \frac{d \mathbf{k}}{k^{2}} \frac{i \mathbf{k v} q\left(\frac{k_{D i}^{2}}{D_{e}}+\frac{k_{D e}^{2}}{D_{i}}\right)-k^{2} k_{D}^{2}(q+\tilde{S})}{k^{4}+k^{2} k_{D}^{2}-\frac{(\mathbf{k v})^{2}}{D_{e} D_{i}}-i \mathbf{k v}\left(\frac{k_{D i}^{2}}{D_{e}}+\frac{k_{D e}^{2}}{D_{i}}\right)-i \mathbf{k v} k^{2}\left(\frac{1}{D_{e}}+\frac{1}{D_{i}}\right)} .
$$

After integration over angular variables one has

$$
\left.U(v)=\frac{q^{2} k_{D}}{\pi} \int_{0}^{\infty} \frac{d x}{x \tilde{v}^{2}\left(\mu_{2}-\mu_{1}\right) d}\left\{\left[i \mu_{1} \tilde{v} \frac{t+d}{t+1}-x(1-\alpha)\right] \ln \frac{\mu_{1}-1}{\mu_{1}+1}-\left[i \mu_{2} \tilde{v} \frac{t+d}{t+1}-x(1-\alpha)\right] \ln \frac{\mu_{2}-1}{\mu_{2}+1}\right]\right\},
$$

where

$$
\begin{gathered}
\mu_{1,2}=\frac{i\left[x^{2}(1+d)+\frac{t+d}{t+1}\right] \pm \sqrt{4 x^{2} d\left(1+x^{2}\right)-\left[x^{2}(1+d)+\frac{t+d}{t+1}\right]^{2}}}{-2 x \tilde{v} d} \\
\tilde{v}=\frac{v}{D_{i} k_{D}}, \quad d=\frac{D_{i}}{D_{e}}, \quad t=\frac{T_{i}}{T_{e}}, \quad \alpha=-\frac{\tilde{S}}{q}
\end{gathered}
$$


At $v=0$

$$
U(0)=-q^{2} k_{D}(1-\alpha)
$$

A typical behavior of the reduced interaction energy

$$
U(\tilde{v})=\frac{U(\tilde{v})-U(0)}{q^{2} k_{D}}
$$

on the dimensionless velocity $\tilde{v}$ is shown on Fig. 6. As is seen the interaction energy is considerably dependent on the sink intensities $S$. Plasma particle absorption by grain results in the existence of the velocity domain in which the interaction energy decreases with the velocity

growth (curves 2-4 on Fig. 6). Notice that in the case of no fluxes through the grain surface $(S=0)$ the interaction energy can be described by the quadratic velocity dependence (curves 1, 5). However, this is not the case for absorbing grains.

Notice that the possibility of the interaction energy decrease with the velocity growth indicates the possibility of particle acceleration in the course of its motion and, thus, it could be a reason of the grain heating. In fact, the force acting on the grain due to the existence of the induced potential is described by the relations

$$
\mathbf{F}(\mathbf{v})=-q \lim _{\mathbf{r} \rightarrow \mathbf{v} t} \frac{\partial \Phi(\mathbf{r}, t)}{\partial \mathbf{r}}
$$

or after the integration over the angular variables

$$
\begin{aligned}
F(v) & =\frac{i q^{2} k_{D}^{2}}{\pi} \int_{0}^{\infty} \frac{d x}{\tilde{v}^{2}\left(\mu_{1}-\mu_{2}\right) d}\left\{2 i \tilde{v} \frac{t+d}{t+1}\left(\mu_{1}-\mu_{2}\right)+\mu_{1}\left[i \mu_{1} \tilde{v} \frac{t+d}{t+1}-x(1-\alpha)\right] \ln \frac{\mu_{1}-1}{\mu_{2}+1}\right. \\
& \left.-\mu_{2}\left[i \mu_{2} \tilde{v} \frac{t+d}{t+1}-x(1-\alpha)\right] \ln \frac{\mu_{2}-1}{\mu_{2}+1}\right\}
\end{aligned}
$$

where $\mu_{1,2}$ is given by Eq. (42).

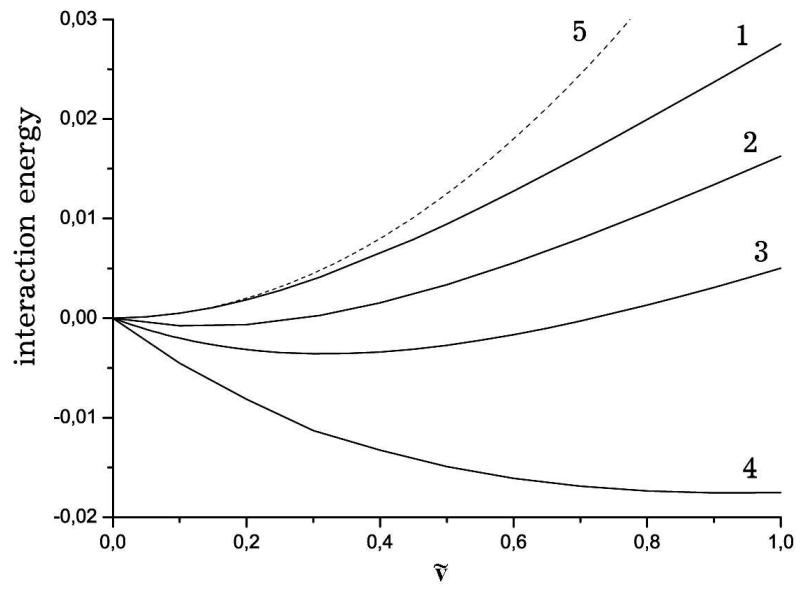

Fig. 6. Dependencies of the reduced energy $U(\tilde{v})$ at $T_{e}=T_{i}, D_{i}=0.01 D_{e}$ for different values of $\alpha ; 1-\alpha=0$, $2-\alpha=0.05,3-\alpha=0.1,4-\alpha=0.2$.

The dependencies of the reduced force $F(\tilde{v}) / q^{2} k_{D}^{2}$ on $\tilde{v}$ are presented on Fig. 7. The curves shown on this figure confirm our assumption about the possibility to observe negative friction, i. e. it can be possible that the friction force is directed along the particle velocity.
It is useful to compare the calculated values of the negative friction force with the friction force generated by the grain collisions with neutral atoms. Estimates made for isothermal plasma used in the typical experiments show that the negative friction can exist.

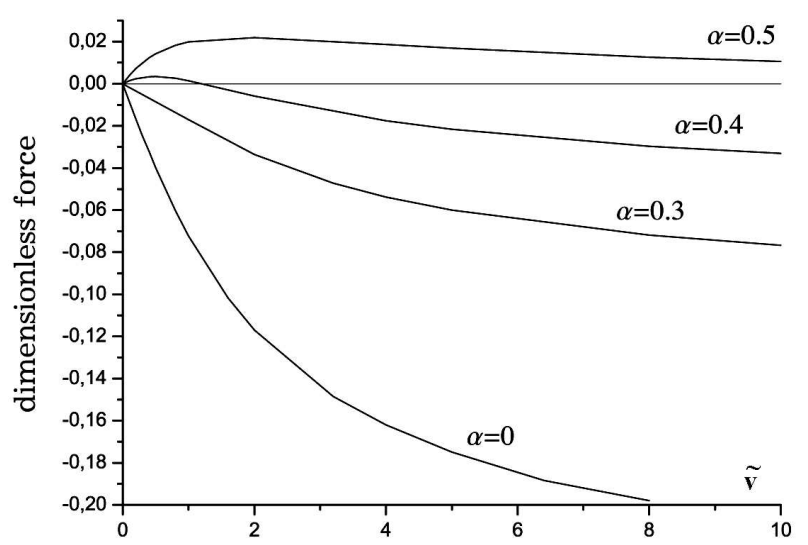

Fig. 7. Dependencies of the dimensionless dynamical friction force $F(\tilde{v}) / q^{2} k_{D}^{2}$ on $\tilde{v}$ at $T_{e}=T_{i}, D_{i}=0.001 D_{e}$ for different values of $\alpha ; 1-\alpha=0,2-\alpha=0.03,3-\alpha=0.4$, $5-\alpha=0.5$. 


\section{CONCLUSIONS}

The model of a point-like sink is proposed to describe the electric grain potential with regard to plasma particle absorption by grain. This makes it possible to obtain analytical solution of the problem of grain screening and to find an explicitly asymptotic behavior of the potential. With the appropriate choice of the parameters of the model (grain charge $q$ and sink intensity $S$ ) the proposed approximation recovers numerical solutions of the consistent nonlinear problems.
The proposed model turns out to be efficient for the description of the screening of the moving grains. Obtained analytical solutions for the grain potentials is used to calculate the grain-plasma interaction energy and dynamical friction force. The calculations show a possibility of the existence of negative friction.

The present work was partially supported by the Program of Fundamental Research of the Department of Physics and Astronomy of the National Academy of Sciences of Ukraine and the Russian Fundation for Fundamental Research (grants 05-02-17258-a and 06-02-08230ofi).
[1] Yu. P. Raizer, Physics of Gas Discharge (Nauka, Moscow, 1987) (in Russian).

[2] V. V. Ivanov, T. V. Rakhimova, A. O. Serov, N. V. Suetin, A. F. Pal', J. Exp. Theor. Phys. 88, 1105 (1999); A. F. Pal', A. N. Starostin, A. V. Filippov, Plasma Phys. Rep. 27, 143 (2001).

[3] A. F. Pal', D. V. Sivokhin, A. N. Starostin, A. V. Filippov, V. E. Fortov, Plasma Phys. Rep. 28, 28 (2002).

[4] T. Bystrenko, A. Zagorodny, Phys. Lett. A 299, 383 (2002).

[5] A. V. Filippov, N. A. Dyatko, A. F. Pal', A. N. Starostin, Plasma Phys. Rep. 29, 190 (2003).

[6] O. Bystrenko, A. Zagorodny, Phys. Rev. E 67, 066403 (2003).

[7] A. G. Leonov, A. F. Pal', A. N. Starostin, A. F. Filippov,
JETP Lett. 77, 482 ( 2003).

[8] A. G. Leonov, A. F. Pal', A. N. Starostin, A. F. Filippov, J. Exp. Theor. Phys. 99, 61 (2004).

[9] A. Zagorodny, V. Mal'nev, S. Rumyantsev, Ukr. J. Phys. 50, 448 (2005).

[10] A. Meltzer, A. Homann, A. Piel, Phys. Rev. E 53, 2757 (1996).

[11] G. Morfill, H. Thomas, U. Konopka, M. Zuzic, Phys. Plasmas 6, 1769 (1999).

[12] O. Bystrenko, A. Zagorodny, Phys. Lett. A 255, 325 (1999).

[13] A. V. Filippov, A. G. Zagorodny, A. F. Pal', A. N. Starostin, JETP Lett. 81, 146 ( 2005).

[14] A. V. Filippov, A. G. Zagorodny, A. I. Momot, A. F. Pal', A. N. Starostin, J. Exp. Theor. Phys. 104, 147 (2007).

\title{
ЕКРАНУВАННЯ МАКРОЧАСТИНКИ У СЛАБОЙОНІЗОВАНІЙ ПЛАЗМІ
}

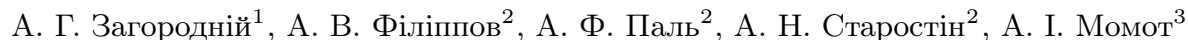 \\ ${ }^{1}$ Інститут теоретичної фізики ім. М. М. Боголюбова, Київ, Украӥна \\ ${ }^{2}$ Троӥчвкий інститут інонваційних та термолдерних досліджень, Тройцьк, Росія \\ ${ }^{3}$ Київсъкий начіональний університет імені Тараса Шевченка, Київ, Украйна
}

\begin{abstract}
Запропоновано новий підхід, який дозволяє описати аналітично ефективні потенціяли порошинок у плазмі. Припускається, що врахування поглинання електронів та йонів порошинкою може здійснюватися за допомогою ефективних точкових стоків у рівняннях, які описують динаміку плазми. Такий підхід дає змогу одержати явні вирази для ефективного потенціялу та розподілу заряду. Показано, що поглинання плазмових частинок порошинкою приводить до появи далекосяжної асимптотики ефективного потенціялу. Розглянуто також динамічне екранування порошинки, що рухається. Розраховано енергію взаємодії порошинки з індукованим нею зарядом та силу, яка діє на порошинку, що рухається. Показано можливість від'ємного тертя.
\end{abstract}

